\title{
A Novel Approach to Output Process Characterization for Token Ring Networks Supporting Priorities*
}

\author{
Mingfu Li Zsehong Tsai \\ Department of Electrical Engineering \\ National Taiwan University, Taipei, Taiwan, R.O.C. \\ Tel: 886-2-3635251, 886-2-3625252 Ext. \\ Fax: 886-2-3638247 \\ E-mail: ztsai@cc.ee.ntu.edu.tw
}

\begin{abstract}
We consider a $L A N$ environment in which two token rings are interconnected via remote bridges. Traffic streams of two priority classes are supported. We present an approach to characterize the output process of one token ring with a bridge node, using a 3-phase Markov Modulated Poisson Process. Our numerical results indicate that this approximated process can be much more accurate than the Poisson Process for the performance analysis of the bridge node.
\end{abstract}

\section{Introduction}

In recent years, significant research has been dedicated to message delay analysis for various token schemes or polling protocols. Basic performance results of such networks are available in [1] and a survey can be found in [2]. Most works assumed messages arriving at a station to belong to a single class and employed a single service discipline, such as exhaustive, gated, or limited. In practice, there may be many kinds of traffic coexisting in a local area network, such as voice, video, data, regular text, etc.. For example, modern integrated-services multiple-access networks and in particular high speed local area networks are typically required to provide "isochronous" and "asynchronous" type services. That is, a network can often be used as a multi-media communication system. Many approaches have been proposed to provide access control for token networks or polling systems which support multi-priority messages. For local area networks, much use has been made of the IEEE 802.4 token bus [3] and 802.5 token ring [4] protocol recommendations, which can support up to 4 and 8 different priority levels, respectively. For fiber-based local area networks, FDDI [5] specifies the use of an IEEE 802 token-bus type priority access scheme over a physical token-ring implementation. Several priority

*This work was supported by National Science Council of the Republic of China under Grant NSC81-0408-E-002-05. access methods have been investigated in $[6],[7],[8]$, $[9],[10]$.

There is always a limit to the number of stations that can be attached to a single local area network. In order to increase the number of stations and to extend the distance covered by the network, local area networks closely located can be interconnected by a bridge or gateway, which is a station with special functions such as routing and store-and-forward operation for internetwork messages. Since internetwork messages from all stations need to pass through the bridge, the latter can easily become the network bottleneck. Such interconnected local area networks are considered in [11], [13]. The basic problem in analyzing interconnected systems is that of characterizing the output process of a local area network. And the output process of a local area network system depends on the protocol that has been employed. Such issues have been treated in [12], [14], [15]. Takine, Takahashi and Hasegawa [12] considered the polling system with a single buffer at each station. They obtained the output process at each station. This result was then applied to the throughput and mean waiting time analyses of an interconnected polling system which consists of several homogeneous stations with single buffers and a depot station (bridge) with infinite buffer. In [14], Bernoulli and first-order Markov processes were used to approximate the output process of a class of slotted multiuser random access communication networks. In [15], the idea of approximating the output process of slotted multiuser random access communication networks by a 2nd-order Markov process was introduced.

In this paper, we consider a system where two token ring networks with only two priority classes are interconnected by two remote bridges (or gateways) and one full-duplex link. Each bridge provides two separate buffers for each transmission direction. To achieve high throughput, the bridges perform only very simple routing and store-and-forward functions, but not involved in error- or flow- control. For most bridges, their filtering and forwarding rates are usually very high, while the most frequently used links operate at $1.544 \mathrm{Mbps}$ (T-1 lines) and $56 \mathrm{kbps}$. Therefore, com- 
paring the bridge processing time with the link transmission time, we find that the network bottleneck is at the bridge output link, especially for the link operating at $56 \mathrm{kbps}$. It indicates that the performance of the bridge node needs to be investigated. Since the output process of the token ring is bursty, we use a 3-phase Markov Modulated Poisson Process (MMPP) to approximate this output process. In this 3-phase MMP$P$, traffic streams of both priority classes are characterized. We then employ the 3-phase MMPP as the arrival process of the bridge node for its performance analysis.

The organization of the paper is as follows. In section 2 we describe the assumptions of our model and the justifications for these assumptions. In section 3 we present an MMPP model for the output process of a token ring network and analyze the busy periods on the ring. In section 4 we analyze the bridge performance. In section 5 we provide some examples. Finally in section 6 we present our conclusions.

\section{Model description}

In this study, we use one model for simulation and a second model for output process characterization. Since these two models are very similar, the following descriptions hold for both models unless stated otherwise.

The local token ring considered here is assumed to contain $g$ infinite buffer stations and one infinite buffer bridge node. And we assume the packet arrival processes at the $i$ th station $(1 \leq i \leq g)$ to follow the Poisson processes with arrival rates $\lambda_{i H}$ and $\lambda_{i L}$ for high and low priority packets, respectively. As to the packet arrival processes from the transmission link to the bridge node, the Poisson property usually does not hold. For simplicity, in the analytical model we still assume that they are and set their arrival rates for high and low priority packets to be $\lambda_{O H}$ and $\lambda_{O L}$, respectively. This assumption is acceptable if the arrival rates $\lambda_{O H}$ and $\lambda_{O L}$ are not too large compared to the total arrival rates $\lambda_{H}$ and $\lambda_{L}$, respectively, where $\lambda_{H}=\sum_{i=0}^{g} \lambda_{i H}$ and $\lambda_{L}=\sum_{i=0}^{g} \lambda_{i L}$. Moreover, the packet service time distributions for both priority classes at each station and the bridge node are assumed to be identical and independent Erlang-K distributions with mean $b$. Usually, the transmission rate for a token ring is $4 \mathrm{Mbps}$ or $16 \mathrm{Mbps}$. It can be shown that the mean packet service time is in the order of mili-second. As to the walk time (due to the bit latency and the propagation delay) from the $i$ th station to the $(i+1)$ th station, since the bit latency is 1 or 2 bits at each station and the bridge node, it is nearly a constant and is usually of only 2 to 5 bit duration in a small ring when the propagation delay is included. Obviously, the walk time can be assumed to be negligible when the packet length is long. Therefore, in the analytical model we also make a zero-walk time assumption. On the link, the packet service times are also assumed to be identical and independent Erlang$\mathrm{K}$ random variables with mean $K / \mu$ for both priority packets. Under the above mentioned assumptions, we can model the remote bridge node on a local token ring as a single server queue with nonpreemptive priority queueing discipline as shown in Fig. 1. For multi-priority traffic, the arrivals from the local ring to the bridge node will be a superposition of several packet streams with general interarrival time distributions and the output link can be modeled as the bridge server. So the general queueing model at the remote bridge node is a $\sum_{i} G_{i} / E_{K} / 1$ nonpreemptive priority queue.

As to the medium access protocol, we employ the procedure defined in IEEE standard 802.5 [4]. According to the standard, for the token ring with only two priority classes, when the token arrives at one station, the station will capture the token and transmit its packets if the priority levels of those packets are not lower than that of the token. Otherwise, the station only makes reservation on the token if it has packets to be transmitted, or just passes the token to the next station. When certain station captures the token and begins transmitting packets, high priority packets may arrive at the other stations. Then reservations can be made for these new arrivals on the transmission packet header in order to determine the priority level of the next released token. In IEEE 802.5 [4], the reservation field resides in the transmission packet header. If we neglect the propagation delay and the bit latency at each station, we have a system in which the stations with packets at the beginning of the current packet transmission could reserve their transmission rights on the reservation field. Besides, the station which upgrades the token priority should downgrade the priority of the token from high to low when alI the high priority packet transmissions are finished. So that the low priority packets can be transmitted. The dwell time that the station can hold the token to transmit packets is called the token holding time. According to the length of the token holding time, we usually have three different dwell-time service disciplines, limited-1 service; exhaustive service; and gated service disciplines. In our model, the service discipline at all stations and bridges are set to be limited-1. For video, audio and important data traffic, we can assign high priority to their packets in such a network to achieve better quality of service.

\section{Process characterization}

In this section, we show how to characterize the departure process of the token ring network, which is just the arrival process at the bridge node. In the first subsection, we map the departure process to a 3-phase Markov Modulated Poisson Process. Then we deduce the transition rates of the MMPP model in terms of system parameters. In the second subsection, we analyze the busy periods and determine all the parameters described in the first subsection.

\subsection{Modeling approach}

The queueing model of an output link at the bridge on a token ring is a generic $\sum_{i} G_{i} / E_{K} / 1$ queue, where 
the interarrival time of the ith traffic class is of a general distribution. However, several assumptions and approximations are required to make the analysis of such queueing model mathmatically tractable. Therefore, an MMPP $/ E_{K} / 1 / N$ model is used in this paper. That is, the superposition of $G_{i}$ streams is approximated by the Markov Modulated Poisson Process (MMPP), and we set $N$ large enough to approximate the infinite buffer assumption. For the token ring network with only two priority traffic classes, one 3-phase MMP$P$ model can be defined using the phase set $\{H, L, I\}$, where the phase $\mathrm{H}$ indicates the ring to be in the busy period with high priority token, $L$ represents the busy period with low priority token, and I denotes the idle period at the token ring network. According to the IEEE 802.5 standard, in phase I the token priority is low.

We use the 3-phase MMPP model as an approximation of the packet arrival process at the bridge node. Fig. 2 shows the MMPP transition diagram. In phase $H$, high priority packets arrive with a rate equal to $\alpha_{s}$ following a Poisson process, but low priority packet arrivals do not occur. In phase L, both high and low priority packets arrive according to Poisson processes with rates equal to $\alpha_{L H}$ and $\alpha_{L}$ respectively. However, in phase $I$, since the system is idle, neither high nor low priority packets will arrive. When the system is idle, the first arrived packet which changes the system state from idle to busy should capture the low priority token and begin its transmission, independent of its priority class. Therefore, there is no transition probability from phase $I$ into phase $H$. Furthermore, since we assume high and low priority packets to arrive at a token ring according to the Poisson processes with total rates $\lambda_{H}, \lambda_{L}$ respectively, the idle period duration is an exponentially distributed random variable with mean $\frac{1}{\lambda_{F}+\lambda_{L}}$. As a result, the transition rate from phase I into phase $\mathrm{L}$ is equal to $\lambda=\lambda_{H}+\lambda_{L}$. Therefore, the generator matrix for denoting the transition rates among phases $\{\mathrm{H}, \mathrm{L}, \mathrm{I}\}$ is given by

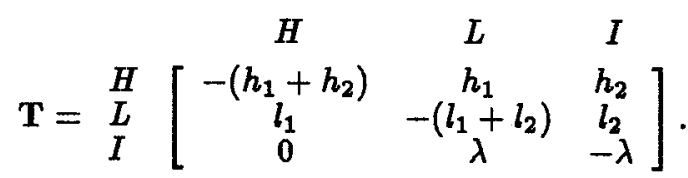

In the following, we derive the parameters which are needed for our MMPP arrival process model. Once they are obtained, we can then proceed to the performance analysis of the bridge. From Fig. 2, the following two global balance equations are readily obtained.

$$
\begin{aligned}
\left(h_{1}+h_{2}\right) P_{H} & =l_{1} P_{L} \\
\left(l_{1}+l_{2}\right) P_{L} & =h_{1} P_{H}+\lambda P_{I},
\end{aligned}
$$

where $P_{H}, P_{L}$, and $P_{I}$ are, respectively, the probabilities that the system lies in phases $H, L$, and $I$ in steady state. Obviously, $P_{H}+P_{L}+P_{I}$ is equal to 1 . And one can easily derive the mean sojourn times of the phases $H, L$, and $I$ to be given by :

$$
\bar{H}=\frac{1}{h_{1}+h_{2}}
$$

$$
\begin{aligned}
& \bar{L}=\frac{1}{l_{1}+l_{2}} \\
& \bar{I}=\frac{1}{\lambda},
\end{aligned}
$$

where $H$ is the random variable representing the length of the busy period with high priority token; $L$ is the random variable indicating the length of the busy period with low priority token; and $I$ is the random variable denoting the length of the idle period.

Solving equations (1)-(4), we have

$$
\begin{aligned}
l_{1} & =\frac{P_{H}}{P_{L} \bar{H}} \\
l_{2} & =\frac{1}{\bar{L}}-\frac{P_{H}}{P_{L} \bar{H}} \\
h_{1} & =\frac{P_{L}}{P_{H} \bar{L}}-\frac{P_{I} \lambda}{P_{H}} \\
h_{2} & =\frac{1}{\bar{H}}-\frac{P_{L}}{P_{H} \bar{L}}+\frac{P_{I} \lambda}{P_{H}} .
\end{aligned}
$$

Next, let the probabilities of high and low priority packets in the local token ring being routed to the remote one be given by $P_{R H}$ and $P_{R L}$, respectively. We assume that packets coming from the remote token ring will not be routed back. Since the mean service times of high and low priority packets are both equal to $b$, and by the flow conservations during phases $H$ and $\mathrm{L}$, the parameters $\alpha_{H}, \alpha_{L}$, and $\alpha_{L H}$ must satisfy the following equations:

$$
\begin{aligned}
\frac{1}{b} & =\frac{\alpha_{H}}{P_{R H}}\left(\frac{\lambda_{H}}{\sum_{i=1}^{g} \lambda_{i H}}\right), \\
\frac{1}{b} & =\frac{\alpha_{L}}{P_{R L}}\left(\frac{\lambda_{L}}{\sum_{i=1}^{g} \lambda_{i L}}\right)+\frac{\alpha_{L H}}{P_{R H}}\left(\frac{\lambda_{H}}{\sum_{i=1}^{g} \lambda_{i H}}\right)
\end{aligned}
$$

Moreover, since all the low priority packets depart from the ring in phase $\mathrm{L}$, one should obtain

$$
\left(\sum_{i=1}^{g} \lambda_{i L}\right) P_{R L}=\alpha_{L} P_{L} \text {. }
$$

That is, the expressions for $\alpha_{H}, \alpha_{L}$ and $\alpha_{L H}$ are given by

$$
\begin{aligned}
\alpha_{H} & =\left(\frac{\sum_{i=1}^{g} \lambda_{i H}}{\lambda_{H}}\right) \frac{P_{R H}}{b} \\
\alpha_{L} & =\left(\sum_{i=1}^{g} \lambda_{i L}\right) \frac{P_{R L}}{P_{L}}, \\
\alpha_{L H} & =\left(\frac{\sum_{i=1}^{g} \lambda_{i H}}{\lambda_{H}}\right)\left(\frac{P_{R H}}{b}-\frac{\lambda_{L} P_{R H}}{P_{L}}\right) .
\end{aligned}
$$

Once the values of $\bar{H}, \bar{L}, P_{H}, P_{L}$, and $P_{I}$ are determined, one can calculate all the parameters for the MMPP model by equations (6)-(9) and (13)-(15). We now proceed to derive close form formulas for these parameters. 


\subsection{Busy period analysis} that

For an $M / G / 1$ queueing system, it is well known

$$
H_{1}^{\star}(s)=B^{\star}\left[s+\lambda_{H}\left(1-H_{1}^{\star}(s)\right)\right],
$$

and it is easy to show that

$$
H_{n}^{\star}(s)=\left[H_{1}^{\star}(s)\right]^{n},
$$

where $H_{n}^{\star}(s)$ is the Laplace-Stieltjes Transform (LST) of the random variable $H_{n}$ which denotes the length of the busy period with high priority token started with $n(n \geq 1)$ number of high priority packets; and $B^{\star}(s)$ is the LST of $B(t)$ which is the CDF of the packet service time distribution for both priority packets on the local token ring. Therefore, the LST of the random variable $H$ can be obtained from the following equation.

$$
H^{\star}(s)=\sum_{n=1}^{\infty} p_{n} H_{n}^{\star}(s)
$$

where $p_{n}$ is the probability that there are $n$ number of high priority packets in the system at the beginning instant of phase $H$, and it is given as below:

$$
\begin{gathered}
p_{n}=\frac{1}{P_{h}} \sum_{m=1}^{n}\left\{a_{m} \int_{0}^{\infty} \frac{\left(\lambda_{H} t\right)^{m}}{m !} e^{-\lambda_{H H} t} d B(t)+\right. \\
\left.\left(1-a_{m+1}\right) \int_{0}^{\infty} \frac{\left(\lambda_{H} t\right)^{m+1}}{(m+1) !} e^{-\lambda_{H} t} d B(t)\right\} \\
\int_{0}^{\infty} \frac{\left(\lambda_{H} t\right)^{n-m}}{(n-m) !} e^{-\lambda_{H} t} d B(t) .
\end{gathered}
$$

The first (second) term in the summation accounts for the condition that the packet served just before the $H$ period is a low (high) priority packet. The parameter $a_{m}$ is the probability that the low priority packet will be served next, given that there are $m$ number of high priority packet arrivals at the present service time, and it is of the form:

$$
\begin{aligned}
a_{m} \cong \sum_{i=0}^{N} \frac{\lambda_{i}}{\lambda} \sum_{j=i+2}^{i} \sum_{p=1}^{m} C_{p}^{m}\left(\frac{\lambda_{j H}}{\lambda_{H}}\right)^{p}\left(\frac{\sum_{k=j+1}^{i} \lambda_{k H}}{\lambda_{H}}\right)^{m-p} \\
\cdot\left\{1-\prod_{k=i+1}^{j-1}\left(1-\lambda_{k L} \bar{c}_{i k}\right)\right\}
\end{aligned}
$$

where the indices $i, j$ are the relative locations on the ring, and $\bar{c}_{i k}$ is the conditional mean cycle time in phase $\mathrm{L}$, given that there is a packet served at station $i$ and no packets served between stations $(i+1)$ to $(k-$ 1). For token ring networks, $\bar{c}_{i k}$ can be approximated as

$$
\bar{c}_{i k} \cong b+\sum_{j \neq i, \cdots, k-1} b\left\{\left(1-\int_{0}^{\infty} e^{-\lambda_{j H} t} d B(t)\right)+\lambda_{j L} \bar{c}_{i k}\right\}
$$

Subsequently, $\overline{\mathbf{c}}_{i k}$ can be written as

$$
\bar{c}_{i k} \cong \frac{b+b \sum_{j \neq i, \cdots, k-1}\left[1-B^{\star}\left(\lambda_{j H}\right)\right]}{1-\sum_{j \neq i, \cdots, k-1} \rho_{j L}} .
$$

As to $P_{h}$ in (19), it is a normalization factor equal to the probability that an arbitrary packet transmission in phase $\mathrm{L}$ leads to a state transition to phase $\mathrm{H}$, and is expressed as

$$
\begin{aligned}
P_{h}= & a_{1} \int_{0}^{\infty} \lambda_{H} t e^{-\lambda_{H} t} d B(t)+ \\
& \sum_{m=2}^{\infty} \int_{0}^{\infty} \frac{\left(\lambda_{H} t\right)^{m}}{m !} e^{-\lambda_{H} t} d B(t) .
\end{aligned}
$$

For most traffic conditions, we find that the terms $a_{m}(m \geq 2)$ are negligible. So from (18) we can approximate the mean value of $H$ as

$$
\begin{aligned}
\bar{H} \cong & \overline{P_{1}}\left\{a_{1}\left(1+\rho_{H}\right) \int_{0}^{\infty} \lambda_{H} t e^{-\lambda_{H} t} d B(t)+\right. \\
& {\left[1-\int_{0}^{\infty} \lambda_{H} t e^{-\lambda_{H} t} d B(t)\right] \rho_{H}-} \\
& \left.\left(1-\rho_{H}\right)\left[1-B^{\star}\left(\lambda_{H}\right)\right]\right\}
\end{aligned}
$$

where $\rho_{H}$ is the utilization of the token for high priority class packets and is equal to $\lambda_{H} b$. And from (16), $\overline{H_{1}}$ is given as

$$
\overline{H_{1}}=\frac{b}{1-\rho_{H}} .
$$

Next, we will derive a formula for the mean value of the busy period $L$. The system time can be combined by $H, L$, and $I$ as shown in Fig. 3, where it shows the only two possible cases between two subsequent high priority token periods $H$. When we set the walk times to be zero, then in steady state, $P_{I}$ which is the probability of the system being idle is equal to $1-\rho$, where $\rho$ is the utilization of the token for both priority packets and is equal to $\lambda b$. For ergodic processes, one can look at the probability $P_{S}$ as the percentage of time in the steady state that the system is in phase $S$, where $S=\mathrm{H}, \mathrm{L}, \mathrm{I}$. Thus, one can show that

$$
\begin{aligned}
P_{H} & =\frac{\bar{H}}{\overline{\bar{H}}+\overline{T_{L}}} \\
P_{I} & =\frac{\overline{N_{I}} \bar{I}}{\bar{H}+\overline{T_{L}}},
\end{aligned}
$$

where $T_{L}$ is the length of the period between two subsequent high priority token periods $\mathrm{H}$; and $N_{I}$ is the number of idle periods in a $T_{L}$ period. In Fig. 3, let the probabilities that the period $\mathrm{H}$ followed by $\mathrm{I}$ and $L$ be equal to $P_{a}$ and $P_{b}$, respectively. Obviously, $P_{a}+P_{b}$ must be equal to 1 . Since $P_{a}$ is equal to the joint probability that there are no low priority packets in the system at the packet departure instant and no 
low priority packet arrivals occur during phase $H$, we can approximate $P_{a}$ as

$$
P_{a} \cong\left(1-\rho_{L}\right) \int_{0}^{\infty} e^{-\lambda_{L} t} d H(t)=\left(1-\rho_{z}\right) H^{\star}\left(\lambda_{L}\right)
$$

where $\rho_{z}$ is the utilization of the token for low priority class packets and is equal to $\lambda_{L} b$, and

$$
P_{b}=1-P_{a} \text {. }
$$

Then according to Fig. 3, we have

$$
\overline{T_{L}}=\left(\overline{N_{I}}+P_{b}\right) \bar{L}+\bar{N}_{I} \bar{I}
$$

The probabilities that the system will change to phase $I$ and phase $H$ at the packet departure instan$t$, given the system in phase $L$, are approximated by $(1-\rho)$ and $P_{h}$, respectively. So the transition probability from phase $L$ into phase $H$ is equal to

$$
P_{L H}=\frac{P_{h}}{P_{h}+(1-\rho)} .
$$

Now, we can calculate $\overline{N_{I}}$ from the following equation:

$$
\overline{N_{I}}+P_{b}=\frac{1}{P_{L H}}
$$

Using equation (27), we can obtain $\overline{T_{L}}$ as follows:

$$
\overline{T_{L}}=\frac{\bar{N}_{I} \bar{I}}{1-\rho}-\bar{H} .
$$

And according to equation (29), we have the following formula:

$$
\bar{L}=\frac{\bar{T}_{L}-\bar{N}_{I} \bar{I}}{\bar{N}_{I}+P_{b}} \text {. }
$$

All the parameters that will be employed for the analysis of an MMPP $/ E_{K} / 1 / N$ model are now available.

\section{Performance analysis at the bridge node}

In order to solve the stationary state probabilities of the MMPP $/ E_{K} / 1 / N$ queue, we define $(n(t), i(t), j(t), k(t), s(t))$ as the system state process and $P(n, i, j, k, s)$ as the stationary state probability, where

$n$ : total number of packets in the system, $0 \leq$ $n \leq N$,

$i$ : the phase in which the state lies, and we let $i$ be equal to 0,1 and 2 for phases $H$, $L$ and I, respectively,

$j$ : the number of low priority packets waiting in the buffer, $0 \leq j \leq n-1$, $k$ : the service class, and $k$ is set to be 1 and 2 for high and low priority packets in service, respectively,

$s:$ the service stage, $s=1,2, \cdots, K$.

From the above definitions, it can be found that $(n(t), i(t), j(t), k(t), s(t))$ is a Markov process. Besides, it can be easily shown that the total number of states is $[3+3 K N(N+1)]$.

The generator matrix for the system state process, denoting as $\mathbf{Q}$ (described in Appendix) satisfies the following equations

$$
\mathbf{Q 1}=\mathbf{0}, \quad \mathbf{I} \mathbf{Q}=\mathbf{0}, \quad \mathbf{\Pi 1}=1,
$$

where 1 represents the vector $[1,1, \cdots, 1]^{T}$ with dimension $[3+3 K N(N+1)]$ and $\Pi$ is a vector of $P(n, i, j, k, s)$ with dimension $[3+3 K N(N+1)]$.

Once $P(n, i, j, k, s)$ is obtained by equation (32) and a corresponding $\mathbf{Q}$ matrix, various performance measures can be calculated. For example, the first moment and second moment of queue length formulas can be derived as follows.

Let the $m$ th moment of queue length for high and low priority packets be denoted as $Q^{(m)}(h)$ and $Q^{(m)}(l)$, respectively. They can be shown to be given by

$$
\begin{aligned}
Q^{(m)}(h) & =\sum_{n=1}^{N} \sum_{i=0}^{2} \sum_{j=0}^{n-1} \sum_{k=1}^{2} \sum_{s=1}^{K}(n-1-j)^{m} P(n, i, j, k, s) \\
Q^{(m)}(l) & =\sum_{n=1}^{N} \sum_{i=0}^{2} \sum_{j=0}^{n-1} \sum_{k=1}^{2} \sum_{s=1}^{K} j^{m} P(n, i, j, k, s)
\end{aligned}
$$

\section{Numerical results}

In this section, several examples are provided to illustrate the accuracy of the analytical results present in previous sections. The parameters used in the simulation model are specified as follows. The traffic conditions and network topology on the two interconnected token rings are identical. The transmission rate is 4 $\mathrm{Mbps}$ for the two token rings. The speed of the transmission link between bridges is set to be one third of the ring speed for simplicity. This assumption also takes into account certain overhead existing in the DS-1 link (1.544 Mbps). The mean packet length is 4 kbytes for both priority packets. The walk time is constant and is set equal to 4 bits or $1 \mu \mathrm{s}$. The routing probabilities, $P_{R H}$ and $P_{R L}$ are both equal to 0.5 .

Figures 4 and 5 show the average busy period lengthes of $H$ and $T_{L}$. We consider two traffic conditions in which the low priority traffic load is set to be $1 / 3$ and $2 / 3$ of the total traffic load, respectively. When the low priority traffic load is dominant, our analytical results are very closed to the simulation statistics with only a few percent of errors. In Fig. 6 , the average queue lengthes of both priority packets 
versus the total traffic load on the link are shown. The second moment behavior of the queue length is shown in Fig. 7. We observe that the MMPP model is much better than the Poisson assumption at medium and heavy traffic load. But when the traffic is lightly loaded, the Poisson assumption is also good. This results can be explained as follows. At light traffic load, the output process of the token ring network is almost determined by the arrival process on the token ring network, which is a Poisson process as we assumed, so that the output process is very close to the Poisson process. But at heavy traffic load, the output process is dominated by the packet transmission time distribution on the ring. When the packet transmission time is close to a constant, e.g. the Erlang-4 distribution used in our examples, the approximation of the output process using a Poisson process does not serve well.

\section{Conclusions}

The token behavior of a token ring network supporting two priorities has been analyzed by analytical methods. Also, we have characterized the output process of token ring networks in an internetworking environment. We have then used this process, which is equivalent to the bridge inflow process, to analyze the bridge performance. The numerical results presented in this paper suggest that when the packet length is not exponentially distributed, the Markov Modulated Poisson Process is a good approximation to the output process of a token ring network. These results may be applied to a LAN internetworking environment in which ATM or DQDB networks are used as the backbone and the packet length is of general distributions. In the future, the performance of a finite buffer bridge with different buffer sharing schemes may be analyzed by using this bridge inflow process.

\section{References}

[1] H. Takagi, Analysis of Polling Systems. The MIT Press, 1985.

[2] H. Takagi, "Queueing Analysis of Polling Models," ACM Computing Survey, vol. 20, no. 1, pp. 5-28, Mar. 1988.

[3] Institute of Electrical and Electronics Engineers, Token-Passing Bus Access Method and Physical Layer Specifications. IEEE Standard 802.4, 1989.

[4] Institute of Electrical and Electronics Engineers, Token Ring Access Method and Physical Layer Specifications. IEEE Standard 802.5, 1989.

[5] American National Standards Institute, Fiber Distributed Data Interface (FDDI)-Token Ring Media Access Control (MAC). ANSI X3.139, 1987.

[6] H. Takagi and M. Murata, "Queueing Analysis of Nonpreemptive Reservation Priority Discipline," ACM SIGCOMM'86, pp. 237-244.
[7] J. Gianini and D.R. Manfield, "An Analysis of Symmetric Polling Systems with Two Priority Classes," Perform. Eval., pp. 93-115, 1988.

[8] B. Gréla-M'Poko, M.M. Ali and J.F. Hayes, “Approximate Analysis of Asymmetric Single-Service Prioritized Token Passing Systems," IEEE Trans. Commun. , vol. 39, no. 7, pp. 1037-1040, July 1991.

[9] Z. Tsai and I. Rubin, "Performance of Token Schemes Supporting Delay-Constrained Priority Traffic Streams," IEEE Trans. Commun., vol. 38, pp. 1994-2003, Nov. 1990.

[10] I.' Rubin and J. C.-H. Wu, "Analysis of an FDDI Network Supporting Multiple-Priority Stations with Single-Packet Buffers," IEEE Milcom'92, pp. $172-176$.

[11] W. Bux and D. Grillo, "Flow Control in LocalArea Networks of Interconnected Token Rings," IEEE Trans. Commun. , vol. COM-33, no. 10, pp. 1058-1066, Oct. 1985.

[12] T. Takine, Y. Takahashi, and T. Hasegawa, "Performance Analysis of a Polling System with Single Buffer and Its Application to Interconnected Networks," IEEE J. Select. A reas Commun., vol. SAC-4, no. 6, pp. 802-812, Sept. 1986.

[13] O.C. Ibe and X. Cheng, "Analysis of Interconnected Systems of Token Ring Networks," Computer Commun., vol. 13, no. 3, Apr. 1990.

[14] I. Stavrakakis and D. Kazakos, "On the Approximation of the Output Process of Multiuser Random-Access Communication Networks," IEEE Trans. Commun., vol. 38, no. 2, pp. 172178, Feb. 1990.

[15] I. Stavrakakis and D. Kazakos, "Performance Analysis of a Star Topology of Interconnected Networks Under 2nd-Order Markov Network Output Processes," IEEE Trans. Commun., vol. 38 , no. 10 , pp. $1724-1731$, Oct. 1990.

[16] H. Saito, M. Kawarasaki, and H. Yamada, "An Analysis of Statistical Multiplexing in an ATM Transport Network," IEEE J. Select. Areas Commun., vol. 9, no. 3, pp. 359-368, Apr. 1991.

[17] L. Kleinrock, Queueing Systems. New York: Wiley, 1976. 


\section{Appendix}

\section{Explicit expression for $\mathbf{Q}$}

In the appendix, we will let the generator matrix for denoting the transition rates among phases $\{H, L, I\}$ be

$$
\begin{aligned}
\mathbf{T} & =\left[\begin{array}{ccc}
-\left(h_{1}+h_{2}\right) & h_{1} & h_{2} \\
l_{1} & -\left(l_{1}+l_{2}\right) & l_{2} \\
0 & \lambda & -\lambda
\end{array}\right] \\
& =\left[\begin{array}{ccc}
-\left(q_{12}+q_{13}\right) & q_{12} & q_{13} \\
q_{21} & -\left(q_{21}+q_{23}\right) & q_{23} \\
q_{31} & q_{32} & -\left(q_{31}+q_{32}\right)
\end{array}\right]
\end{aligned}
$$

With the help of the state transition diagram, the generator matrix $Q$ for the 3-phase MMPP $/ E_{K} / 1 / N$ is given by the following :

$$
\mathbf{Q}=\left[\begin{array}{cccccc}
D(0) & \Lambda(1) & 0 & \cdots & \cdots & 0 \\
M(1) & D(1) & \ddots & \ddots & & \vdots \\
0 & M(2) & \ddots & \ddots & \ddots & \vdots \\
\vdots & \ddots & \ddots & \ddots & \Lambda(N-1) & 0 \\
\vdots & & \ddots & \ddots & D(N-1) & \Lambda(N) \\
0 & \ldots & \cdots & 0 & M(N) & D(N)
\end{array}\right]
$$

The detailed submatrices of $\mathbf{Q}$ can be expressed as follows, where the representations of the submatrices $\Lambda, D$, and $M$ follow the convention used in [16].

$$
\begin{aligned}
& \Lambda(1)=\left[\begin{array}{llll}
a_{1,1} & a_{1,2} & \cdots & a_{1,6 K} \\
a_{2,1} & a_{2,2} & \cdots & a_{2,6 K} \\
a_{3,1} & a_{3,2} & \cdots & a_{3,6 K}
\end{array}\right] \text {, } \\
& \text { where } \quad a_{i, j}=\left\{\begin{array}{cl}
\alpha_{H}, & i=1, j=1 \\
\alpha_{L H}, & i=2, j=2 K+1 \\
\alpha_{L}, & i=2, j=3 K+1 \\
0, & \text { otherwise }
\end{array}\right. \\
& \Lambda(k)=\left[\begin{array}{ccc}
\Lambda_{h H} & 0 & 0 \\
0 & \Lambda_{h L} & 0 \\
0 & 0 & 0
\end{array}\right]_{6 K(k-1) \times 6 K k}, k=2, \cdots, N \\
& \Lambda_{n H}=\left[\begin{array}{cccccc}
A_{H} & 0 & \cdots & 0 & 0 & 0 \\
0 & A_{H} & \ddots & \vdots & \vdots & \vdots \\
\vdots & \ddots & \ddots & \ddots & \vdots & \vdots \\
0 & \cdots & 0 & A_{H} & 0 & 0
\end{array}\right]_{2 K(n-1) \times 2 K k}
\end{aligned}
$$

$$
\Lambda_{h L}=\left[\begin{array}{cccccc}
A_{L H} & 0 & A_{L} & 0 & \cdots & 0 \\
0 & A_{L H} & 0 & A_{L} & \ddots & \vdots \\
\vdots & \ddots & \ddots & \ddots & \ddots & 0 \\
0 & \cdots & 0 & A_{L H} & 0 & A_{L}
\end{array}\right]_{2 K(h-1) \times 2 K k}
$$$$
\text { where }\left\{\begin{array}{l}
A_{H}=\operatorname{diag}[\overbrace{\alpha_{H}, \cdots, \alpha_{H}}^{K}] \\
A_{L H}=\operatorname{diag}[\overbrace{\alpha_{L H}, \cdots, \alpha_{L H}}^{K}] \\
A_{L}=\operatorname{diag}[\overbrace{\alpha_{L}, \cdots, \alpha_{L}}^{K}]
\end{array}\right.
$$$$
D(0)=\left[\begin{array}{lll}
d_{01} & q_{12} & q_{13} \\
q_{21} & d_{02} & q_{23} \\
q_{31} & q_{32} & d_{03}
\end{array}\right]
$$

where $\left\{\begin{array}{l}d_{01}=-\left(q_{12}+q_{13}+\alpha_{H}\right) \\ d_{02}=-\left(q_{21}+q_{23}+\alpha_{L H}+\alpha_{L}\right) \\ d_{03}=-\left(q_{31}+q_{32}\right)\end{array}\right.$

$$
\begin{aligned}
& D(k)=\left[\begin{array}{lll}
D_{k 1} & Q_{12}^{k} & Q_{13}^{k} \\
Q_{21}^{k} & D_{k 2} & Q_{23}^{k} \\
Q_{31}^{k} & Q_{32}^{k} & D_{k 3}^{k}
\end{array}\right]_{6 K k \times 0 K h}, k \leq N \\
& Q_{i j}^{k}=\left[\begin{array}{cccc}
q_{i j} & 0 & \cdots & 0 \\
0 & q_{i j} & \ddots & \vdots \\
\vdots & \ddots & \ddots & 0 \\
0 & \cdots & 0 & q_{i j}
\end{array}\right]_{2 K k \times 2 K k},
\end{aligned}
$$

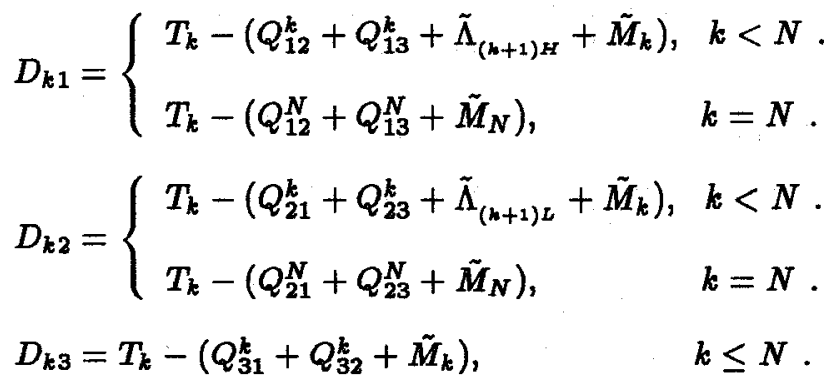$$
\text { where }\left\{\begin{array}{l}
\tilde{M}_{k}=\operatorname{diag}[\overbrace{\mu, \mu, \cdots, \mu}^{2 K k}]_{, k} \tilde{\Lambda}_{(k+1) H}=\operatorname{diag}[\overbrace{\alpha_{k H}, \alpha_{k H}, \cdots, \alpha_{k}}^{2 K k}], \\
\tilde{\Lambda}_{(k+1) L}=\operatorname{diag}[\overbrace{\alpha_{L H}+\alpha_{H}, \cdots, \alpha_{L H}+\alpha_{L}}^{2 K}]
\end{array}\right.
$$

and

$$
T_{k}=\left[\begin{array}{cccc}
t_{1,1} & t_{1,2} & \cdots & t_{1,2 K k} \\
t_{2,1} & t_{2,2} & \cdots & t_{2,2 K k} \\
\vdots & \vdots & \ddots & \vdots \\
t_{2 K k, 1} & a_{2 K k, 2} & \cdots & t_{2 K k, 2 K k}
\end{array}\right]
$$


with

$t_{i, j}= \begin{cases}\mu, & \text { all } i \neq K, 2 K, \cdots, 2 k K, j=i+1 \\ 0, & \text { otherwise }\end{cases}$

$M(k)=\left[\begin{array}{ccc}M_{k} & 0 & 0 \\ 0 & M_{k} & 0 \\ 0 & 0 & M_{k}\end{array}\right]_{\operatorname{Sxk\times \operatorname {Sx}(h-1)}}, k=1, \cdots, N$

$M_{1}=\left[\begin{array}{c}m_{1} \\ m_{2} \\ \vdots \\ m_{2 K}\end{array}\right]$, where $m_{i}= \begin{cases}\mu, & i=K, 2 K \\ 0, & \text { otherwise }\end{cases}$

$M_{2}=\left[\begin{array}{cc}M_{10} & 0 \\ 0 & M_{10}\end{array}\right]_{6 \times \times 2 \pi}$

$M_{k}=\left[\begin{array}{ccc}M_{10} & 0 & 0 \\ 0 & S_{k} & 0 \\ 0 & 0 & M_{10}\end{array}\right]_{2 K n \times 2 \times(n-1)} \quad, k=3, \cdots, N$

$S_{k}=\left[\begin{array}{cccc}S & 0 & \cdots & 0 \\ 0 & S & \ddots & \vdots \\ \vdots & \ddots & \ddots & 0 \\ 0 & \cdots & 0 & S\end{array}\right]_{2 K(h-2) \times 2 K(k-2)}$

where $\left\{\begin{array}{l}M_{10}=\left[M_{1}, 0, \cdots, 0\right]_{2 K \times K}, \\ S=\left[0, M_{10}\right]_{2 \times \times 2 K}\end{array}\right.$

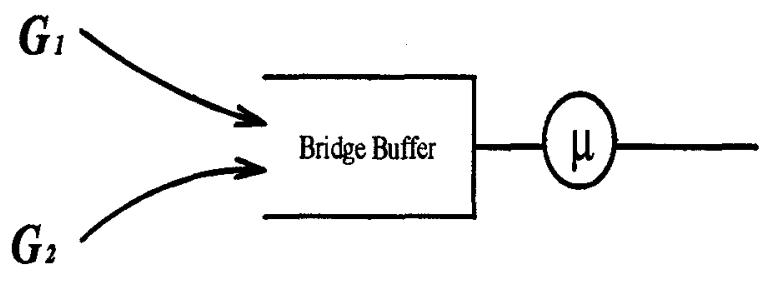

Figure 1: The queueing model of the remote bridge ( $G_{1}$ for high priority stream and $G_{2}$ for low priority stream, $\mu$ is the link service rate).

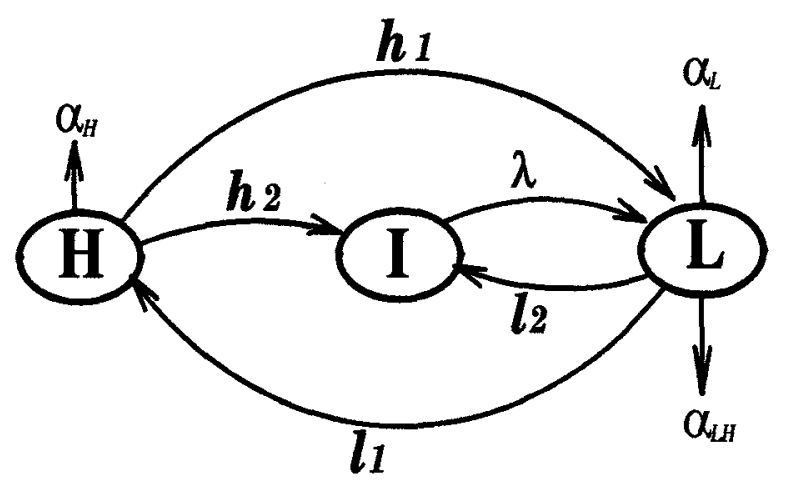

Figure 2: The MMPP transition diagram

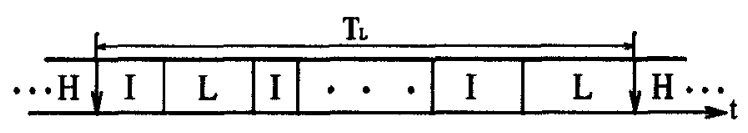

(a)

\begin{tabular}{c}
$T_{L}$ \\
\hline
\end{tabular}

(b)

Figure 3: Two possible sequences of I-L periods between two subsquent periods $H$. 


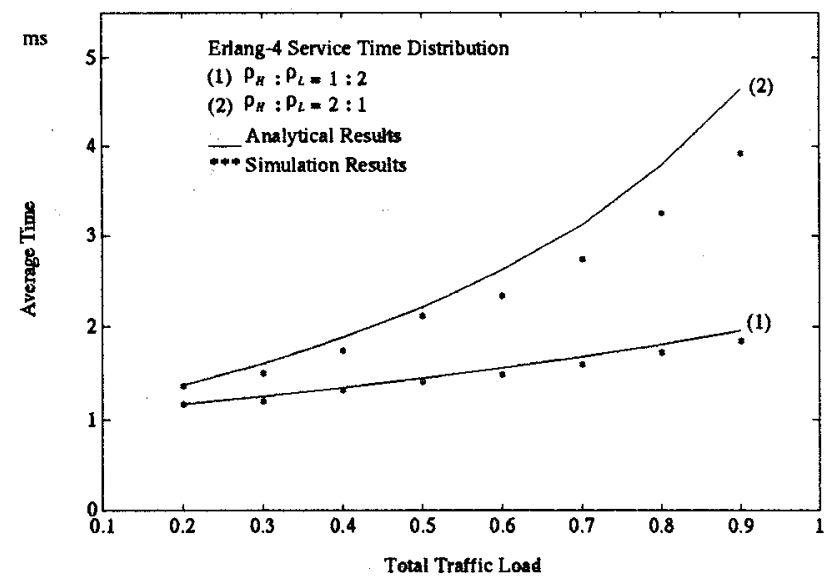

Figure 4: Average busy period length of the high priority token versus total traffic load.

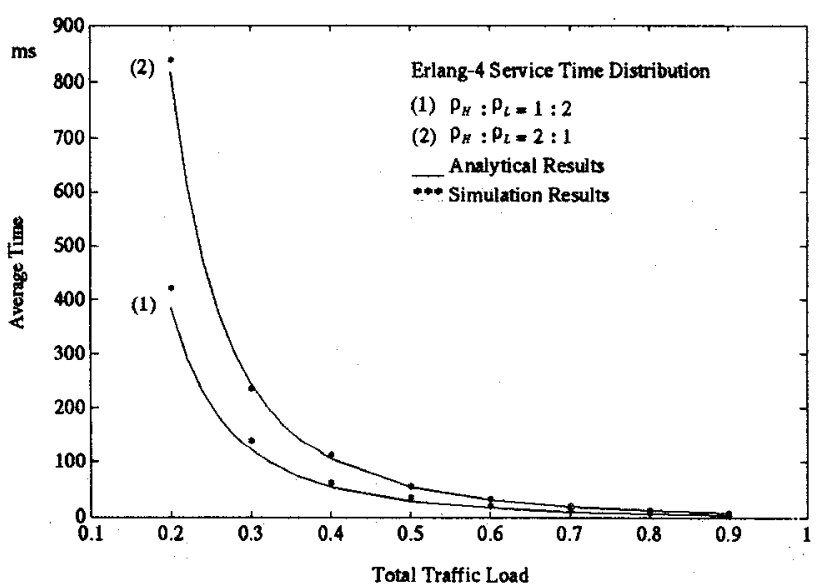

Figure 5: Average busy period length of the low priority token versus total traffic load.

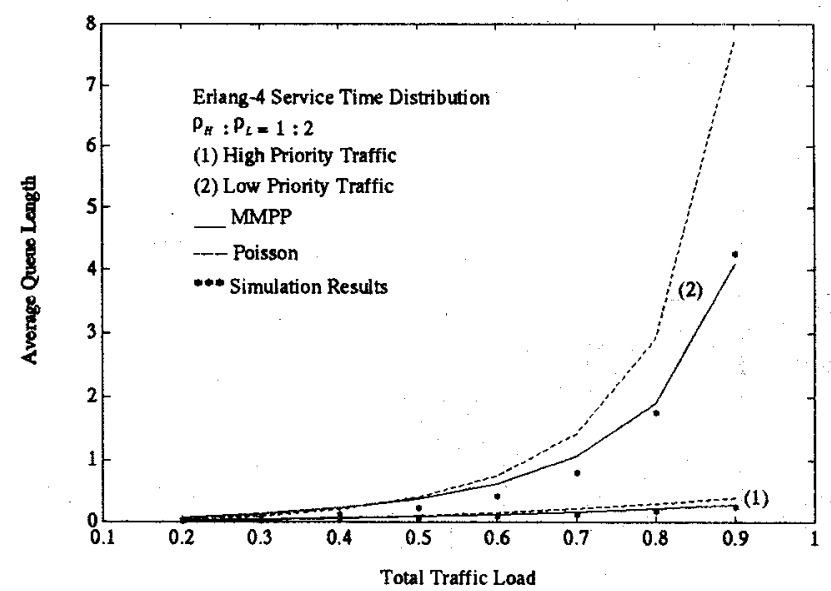

Figure 6: First moment of the queue length at the bridge node.

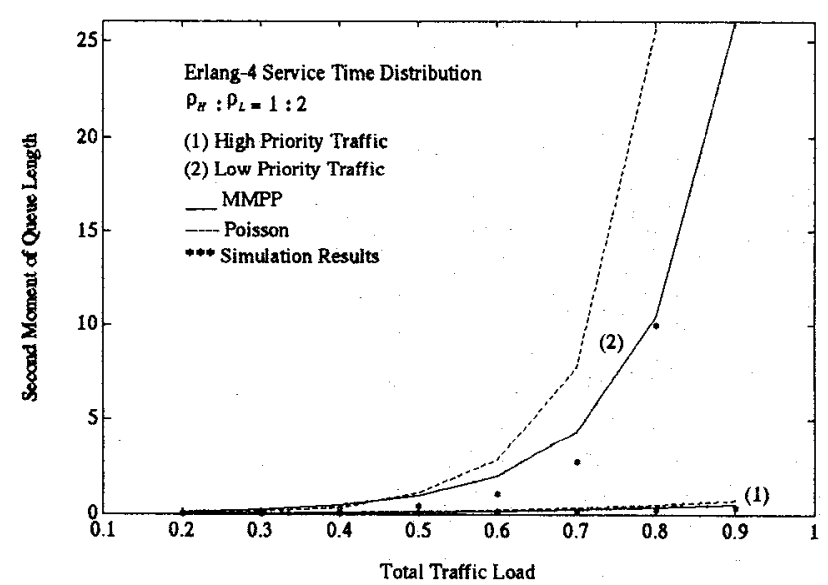

Figure 7: Second moment of the queue length at the bridge node. 